\title{
Therapeutic Targets on Toxoplasma gondii Parasite in Combatting Toxoplasmosis
}

\author{
Sharif Alhassan Abdullahi ${ }^{1,2}$, Ngah Zasmy Unyah ${ }^{1}$, Noshariza Nordin ${ }^{3}$, \\ Rusliza Basir ${ }^{4}$, Wana, Mohammed Nasir ${ }^{1,5}$, Ashraf, Ahmad Alapid ${ }^{1,6}$, \\ Yahaya Hassan $^{1,2}$, Tijjani, Mustapha ${ }^{1,7}$ and Roslaini Abd Majid ${ }^{1^{*}}$ \\ ${ }^{1}$ Department of Medical Microbiology and Parasitology, Faculty of Medicine and Health Sciences, \\ University Putra Malaysia, 43400 Selangor, Malaysia. \\ ${ }^{2}$ College of Health Sciences, Bayero University Kano, P.M.B. 3011, Zaria Road, Kano State, Nigeria. \\ ${ }^{3}$ Department of Biomedical Sciences, Faculty of Medicine and Health Sciences, University Putra \\ Malaysia, 43400 Selangor, Malaysia. \\ ${ }^{4}$ Department of Human Anatomy, Faculty of Medicine and Health Sciences, University Putra \\ Malaysia, 43400, Selangor, Malaysia. \\ ${ }^{5}$ Department of Biological Sciences, Faculty of Sciences, Abubakar Tafawa Balewa University Bauchi, \\ Nigeria. \\ ${ }^{6}$ Department of Zoology, Faculty of Science-Alassaba, University of Gharyan, Libya. \\ ${ }^{7}$ Department of Biological Sciences, Faculty of Science, Yobe State University, Nigeria.
}

Authors' contributions

This work was carried out in collaboration among all authors. All authors read and approved the final manuscript.

Article Information

DOI: $10.9734 / A R R B / 2019 / v 32 i 230081$ Editor(s):

(1) Dr. Oluyomi A. Sowemimo, Department of Zoology, Obafemi Awolowo University, Nigeria. (2) Dr. George Perry, Dean and Professor of Biology, University of Texas at San Antonio, USA. Reviewers:

(1) J. Y. Peter, University of Abuja, Nigeria. (2) Meer Ahmad Mydin Meera, Meer Ahmad Health-Care Consultancy, Malaysia. Complete Peer review History: http://www.sdiarticle3.com/review-history/49444

Review Article

Received 05 April 2019

Accepted 22 June 2019

Published 29 June 2019

\section{ABSTRACT}

The status of toxoplasmosis management is challenged by drug intolerance, compromised efficacy and potential development of drug resistance. However, currently, there are very limited targets on $T$. gondii that serve as the site of action of current medications. This review aimed to explore more potential targets that are essential to parasite survival and are absent in humans. Many unexplored 
targets on T. gondii exist and their specificities to the parasite make them ideal drug targets. The present review had searched relevant English databases such as PubMed, Scopus, Google scholar and Science Direct, for relevant literature on the therapeutic target of $T$. gondii. Many of the enzyme systems in several pathways are essentially palatable as drug targets. Establishing compounds that can target these enzymes on $T$. gondii will greatly be beneficial in treating acute and chronic toxoplasmosis in humans.

Keywords: Toxoplasmosis; T. gondii; essential; drug; targets; enzymes.

\section{INTRODUCTION}

Toxoplasmosis is distributed worldwide and affects more than a third of the world population [1]. The prevalence of this infection differs between regions, countries and even within a country largely due to climatic factors, feeding habits of the populace, availability of the intermediate hosts that include all mammals and birds, and anthropogenic factors [2]. Human transmission is through consumption of infected meat and contaminated water, fruits, and vegetables following soil contamination with the sporulated oocyst shed by the definitive host, the cats [2]. Toxoplasma gondii has a unique affinity to form a chronic course of infection in the brain, retina, placenta, heart and skeletal muscles cells that can lead to chorioretinitis, encephalitis, focal cerebral lesions and congenital malformation in a fetus infected through the pregnant-mother $[1,3]$. The severity of these clinical conditions partly depends on the immune status of the individuals and the strain of $T$. gondii that caused the infection $[1,4]$.

Generally, the current treatment available for toxoplasmosis using pyrimethamine in combination with sulfadiazine as first-line therapy have a lot of adverse effects that range from hematological and liver function derangement to skin manifestation of hypersensitivity reactions $[1,5]$. The alternative therapy that involves the use of macrolides, atovaquone, dapsone and cotrimoxazole, however, have low efficacy, side effects and can be teratogenic, thus limiting their use as anti-toxoplasma drugs.

Though several attempts are ongoing to have new drug candidates against human toxoplasmosis, drug target is only suitable if it fulfills certain criteria that; the it must be essential for parasite growth and survival and must be specific to the organism with no homology in human. These criteria, if strictly adhered to, in drug development against $T$. gondii, issues related to drug toxicity, immune evasion, and economic loss in the agricultural sector will be resolved [6]. In this review, therefore, the potential drug targets that are essential to $T$. gondii survival and specific to the parasite are discussed.

\section{DRUG TREATMENT OF TOXO- PLASMOSIS}

In practice today, drug treatment of toxoplasmosis, though may not be required its nonspecific symptoms manifestation. Apart from their limited number in use, the drugs are generally nonspecific to $T$. gondii and only affect the replicating tachyzoite stage through inhibition of folic acid biosynthesis and protein synthesis. The effect of the drugs and action by the host immune system facilitate rapid stage conversion from the tachyzoite stage to slowly dividing tissue cyst. Most of the drugs do not act on the tissue cyst and cannot cross the biological barriers such as the blood brain barrier [5]. These factors, favor T. gondii intracellular survival in tissues and by extension, favor its possible reactivation and long-term complications.

For decades, the drugs regimen for human toxoplasmosis, include sulfadiazine, pyrimethamine, and atovaquone that have activity against Plasmodium parasites. Others are spiramycin, azithromycin, and clindamycin [7]. To avoid the risk of teratogenicity and development of congenital toxoplasmosis, spiramycin is given to pregnant women who are seropositive. The drug was assessed to reduce complications in utero and in infants after birth [8] and is reliably used in the early phase of toxoplasmosis in pregnant-mother to prevent fetal transmission [9]. However, a prior report by Habib [10], indicated that more than half of the pregnant women who received spiramycin treatment still contain the parasite's DNA in their blood. Therefore, to prevent obstetric complications such as intrauterine growth restriction, intrauterine fetal death, abortion, stillbirth, and congenital toxoplasmosis, treatment of seropositive mother becomes necessary using spiramycin [7]. Combination of trimethoprim/sulfamethoxazole is 
given as prophylaxis in HIVIAIDs patients to inhibit dihydrofolate reductase, (DHFR) and dihydropteroate synthase (DHPS) respectively. Treatment as prophylaxis for those with HIV infection, depending on their CD4+ counts $(<200$ cell/ul), prevents sequelae of reactivation of the latent stage [11]. Atovaquone is an antiplasmodium drug that targets cytochrome bc1 complex which is necessary for mitochondrial electron transport function. It was shown to selectively act on $T$. gondii using cytochrome bc1 as its target. Though it has little or no side effect on the host cell and is effective against acute toxoplasma encephalitis in combination with pyrimethamine or sulfadiazine [12], atovaquone has a very low bioavailability [13]. Atovaquone was also reported to have an effective synergism with sulfadiazine in $\mathrm{RH}$ type 1 strain-infected mice [14].

Treatment failure of the available drugs may be due to prolonged usage. However, due to the complex life cycle of the parasite, possible development of resistance [15], treatment failure in toxoplasma chorioretinitis, encephalitis and congenital infections is on the increase and still under investigation [3]. Furthermore, treatment failure may not only be due to resistance developed by the parasite, but rather, multifactorial causes. Another factor that may cause treatment failure is malabsorption which leads to poor bioavailability of the drugs. In patients with comorbidities, factors such as drugdrug interaction may contribute to treatment failure. Associated with treatment failure is development of resistant or low susceptible strains of the parasite when exposed to a longterm prophylactic or maintenance treatment $[3,16]$.

\section{DRUG RESISTANCE IN T. gondii INFECTION}

Like any other microorganism, drug resistance in the apicomplexan protozoan has been reported $[3,17]$. The possibility of drug resistance is suspected to be responsible for certain treatment failures in patients with acute infections and those with relapse after maintenance therapy [5]. Due to the usually asymptomatic nature of $T$. gondii infection in humans, drug resistance cannot be easily determined because the agent's stages of infection cannot be easily isolated in human $[1,5]$. However, alteration in genes coding for the common enzyme targets, DHFR, and DHPS was found to be the basis for resistance to common drugs treatment in Plasmodium falciparum and Pneumocystis carinii [3]. A study by Aspinal et al. [16], described a sulfonamideresistant isolate of $T$. gondii from clinical cases of human toxoplasmosis. Results from the study showed that sequence alteration in the gene coding for DHPS enzyme confers resistance in $T$. gondii and related parasite $P$. falciparum to sulphonamides. However, in vitro model of experiments has been at the forefront in assessing the drug resistance by $T$. gondii. McFadden et al. [18] provided a suitable suggestion that will help researchers to develop new agents through modification of structural arrangement which will make the drug more effective. Many susceptible, as well as resistant strains of $T$. gondii, were developed in vitro through chemical mutagenesis to assess the level of resistance $[16,19]$. The findings from such studies can help to better design a drug candidate that may have multiple target against the resistant strain of the parasite. Furthermore, the susceptibility of different genotypes of the $T$. gondii has been evaluated. Even though there were no much difference in susceptibility among the genotypes, the highly virulent type I strain had however, low susceptibility in comparison to others $[5,20]$.

\section{POTENTIAL THERAPEUTIC TARGETS IN T. gondii}

The apparent inability of the current drug regimen to act on the tissue cyst containing the slowly replicating bradyzoite in vital tissues, slows the progress of achieving full treatment coverage. To achieve complete treatment of toxoplasmosis, drugs that can be used against different targets, need to be thoroughly explored, identified, and developed. However, the drugs should be specific to T. gondii with minimal or no adverse effects on the host, and active against different stages of the parasite. Many enzymatic activities of $T$. gondii also need to be taken into consideration in that; certain drug molecules can act to inhibit certain biosynthetic pathways thereby inhibiting parasite growth as exemplified by pyrimethamines and sulphonamides. It is essential to develop and administer a drug having reliably understood its mechanism of action, specific target site and the consequences of its action on the parasite and the host. This basically calls for screening procedures to assess the effect of the drug on the parasite biology. Such screening procedures to determine the effect of a potential drug candidate on proliferation inhibition on whole organism or target-based, can be done in vitro or in vivo. 
While the latter is associated with ethical procedures and therefore time consuming and expensive, the former is proved to be easy, simple and inexpensive to carry out when handled by a laboratory expert [21].

\subsection{Protein Kinases}

The protein kinases (PKs) in T. gondii participate in many biological processes of the parasite life cycle [22,23]. The serine-threonine kinases present in $T$. gondii have a catalytic domain containing a glycine rich N-terminal ATP-binding pocket in addition to a central conserved aspartic acid residue required for their catalytic activities $[23,24]$. A report by Piexoto et al. [25] indicated that, T. gondii encodes for 108 PKs genes, that have a high tendency of exhibiting catalytic activities and encodes for pseudo kinase genes that have no catalytic domain. Most of these genes identified, have no orthologues in human, but they are well conserved in apicomplexan parasites like Plasmodium species, prokaryotes and plants. This feature makes the PKs suitable to be used as a drug target for drug discovery using those inhibitors that can specifically target the parasite and consequently with little or no side effect on the host $[23,26,27]$. Both PKs have several functions that are important for parasite survival such as host cell adherence, gliding motility, the formation of the parasitophorous vacuole (PV), intracellular replication, egress as well as modification of host cell microenvironment [24]. The essential PKs in T. gondii that are suitable for drug discovery are herein reviewed.

\subsubsection{Calcium dependent protein kinases (CDPK)}

T. gondii possesses series of CDPKs (Fig. 1) that are essential for various functions that are associated with intracellular calcium ion $\left(\mathrm{Ca}^{2+}\right)$ level [27] that leads to the secretion of microneme proteins essentials for host cell invasion, gliding motility and egress. The CDPKs that are conserved among the apicomplexan parasites include CDPK1, CDPK2, CDPK3, CDPK4, CDPK5, CDPK6, and CDPK7 and their physiological role for parasite survival was studied [28]. The CDPK1 plays a critical role in host cell adhesion, invasion, and egress as proved by the various knockout study as well as developed chemical inhibitors. Several studies reported that activities of CDPK1 was inhibited following exposure to inhibitors that selectively target it sparing mammalian host cell. These inhibitors that were used against CDPK1 include bumped kinase inhibitors [26, 29], ATP-competitive inhibitor [30] and Benzoylbenzimidazole-based selective inhibitors [31].

The most striking feature that makes it suitable for use as a drug target is that it contains a glycine gatekeeper residue situated adjacent to ATP-binding pocket against which many chemical inhibitors were developed. This feature differentiates it with other CDPKs within the parasite, other apicomplexans and that of the mammals as it was shown to be a target by bumped kinase inhibitors in vivo [26] and conditional knockout of CDPK1 was reported to inhibit microneme secretion [32].

Chen et al. [33] reported the development of humoral and cellular immune response among mice that were challenged with $\mathrm{RH}$ tachyzoite after vaccination with plasmid pVAX-CDPK2 vaccine. The mice ware also observed to have amylopectin accumulation, CDPK2 is also a potent candidate for vaccine development. A prior study by Zhang et al. [34] indicated that CDPK2 is essential for amylopectin metabolism in that its deficiency leads to accumulation of starch that leads to the death of $T$. gondii. The CDPK3 was reported to be essential in egress of the tachyzoites [35]. Forward genetic experiment reported by Garrison et al. [36] revealed that CDPK3 plays a role in egress and latent stage conversion in mice. Morlon-Guyot et al. [28] reported that CDPK7 is important in cell division and survival. This report followed a knock-down experiment in which deficiencies in tachyzoite division and growth were due to defects on centrosome integrity. This, according to the study causes restriction in cell division within the vacuoles and impairment of endodyogeny. In addition, Wang et al. [24] reported that CDPK7 might be involved in immune evasion in that there was an increase expression of CDPK7 gene in IFN- $\gamma$ knocked out mice with no difference in expression obtained in vitro using HFF cell. A number of these CDPKs in Plasmodium parasites has been studied and functions were reported to be matched with their orthologues in T. gondii [24]. 


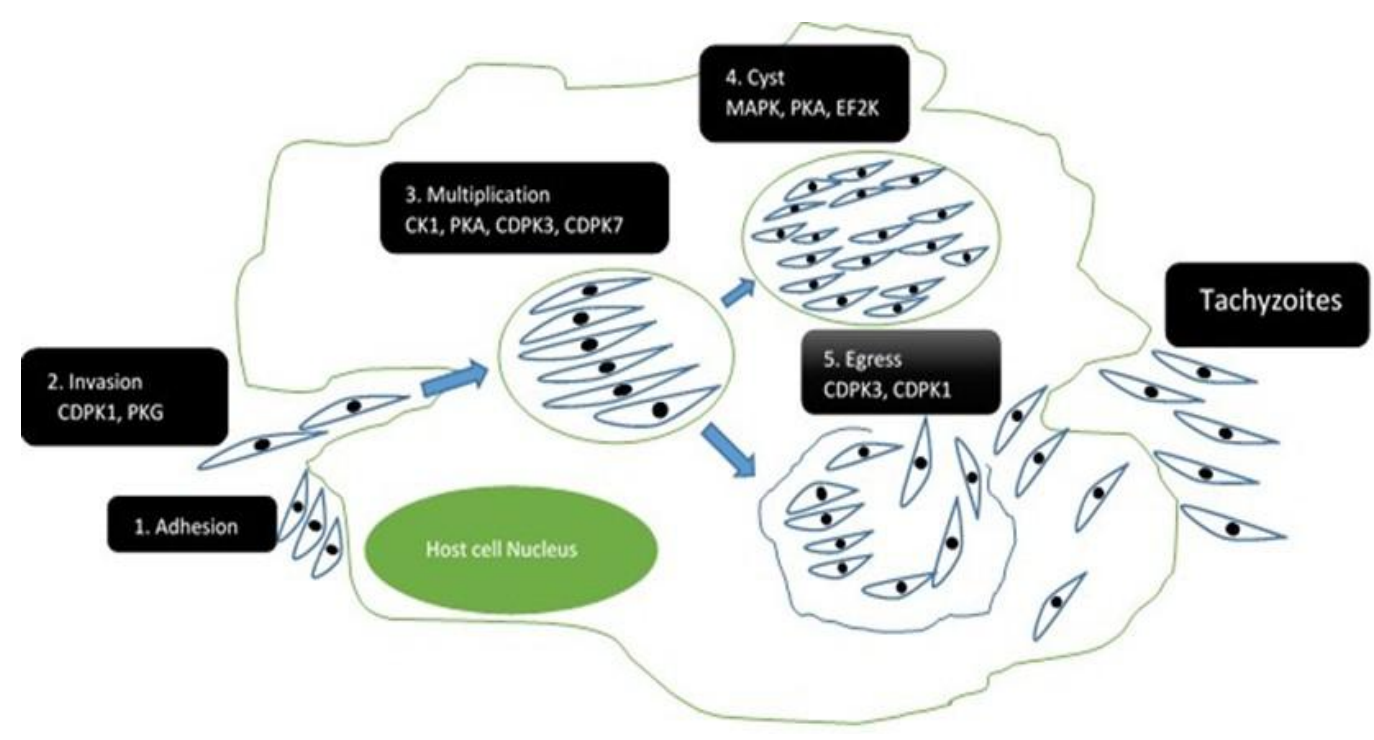

Fig. 1. Toxoplasma protein kinases in the parasite lytic cycle

The CDPK1 and PKG1 participate in adhesion and invasion steps where they cause secretion of microneme proteins. CDPK3 and CDPK1 are also reported to act during egress. PKA-C, CDPK3, CDPK7 and CK1 are involved in cell cycle regulation and growth. MAPK1, PKA-C and eukaryotic initiation factor-2 kinase (EIF2K) participate in stress responses and stage conversion from tachyzoite to bradyzoite (adapted and modified: Wei et al. 2013)

\subsubsection{Casein kinase 1 (CK 1)}

The CK 1 is an essential enzyme in signaling pathways among the serine-threonine protein kinases in eukaryotes that mediates many biological and physiological process in the cells [37]. Though, the function of this kinase group has not been ascertained [23] in T. gondii, their special function in organisms such as DNA repair, cell cycle regulation as well as cytokinesis, can be exploited as potential drug target in $T$. gondii since their role in another related protozoan, Leishmania major, has been reported [38]. Inhibitors such as purvanolol $B$ and aminopurvanolol A were observed to selectively inhibit the $\alpha$-isoform of TgCK1 thereby inhibiting the parasite growth intracellularly while sparing the host cell [39].

\subsubsection{Mitogen-activated protein kinase (MAPK)}

MAPK is an enzyme that regulates some essential cellular activities in $T$. gondii. These activities include regulation of proliferation, stage differentiation and response to stress by the parasite. Its activities are regulated by intracellular calcium level [23]. Activity of MAPK was reported by Wei et al. [40] to be inhibited by pyridinylimidazole that causes morphological changes on the parasite which suggests that the effect of the inhibitor might have caused defects in the parasite's intracellular replication. This inhibitor was also reported to block TgMAPK-1 autophosphorylation [41] thereby reducing its virulence role in toxoplasmosis [42]. For the two isoforms of MAPKs (TgMAPK-1 \& TgMAPK-2) present in $T$. gondii, presence of the threonine, aspartic acid and tyrosine motifs that define their essential role as well as uniqueness from mammalian MAPKs make them one of those PKs that can be exploited as good targets for the development of new drugs against the $T$. gondii $[42,43]$.

\subsection{4 cGMP-dependent protein kinase (cGMP- PKG)}

The cGMP-PKG is one of the PKs involved in microneme secretion that plays a role in hostparasite interaction [23]. It was reported to be an essential protein in $T$. gondii that can be used as a potential drug target against which inhibitors can be developed. Following an in vivo study of a trisubstituted pyrol also known as compound 1; 4-[2-(4-flourophenyl)-5-(1-methylpiperidine-4-yl)$1 \mathrm{H}$-pyrrol-3-yl] pyridine, its cytostatic activity on the $T$. gondii tachyzoite was observed [44]. The same activity was also reported against another 
apicomplexan parasite Emeria tenella [45]. But with the fact that compound 1 is only cytostatic on the parasite, other pathways that regulate microneme secretion may come into play and thus there is still a need to have agents that can completely kill, preferably, the parasite stage in the likes of intracellular parasites equipped with immune evasion mechanism [46].

\subsection{5 cAMP-dependent protein kinase (cAMP- PKA)}

The cAMP-PKA is one of the important PKs regulating many cellular processes in the eukaryotes [47]. Sugi et al. [48] studied the effect PKA on stage interconversion of tachyzoite to bradyzoite asexual stages of $T$. gondii. The study revealed that the catalytic subunit of $T$. gondii PKAc3 that resides within the cytosol and which is specific to coccidian parasites, is responsible for the maintenance of the tachyzoite stage during infection. This was possible despite exposure to certain stress factors that could have triggered differentiation to bradyzoite hence the cystic stage, as demonstrated in vivo [48]. Therefore, developing a potent drug against PKAc3, will greatly help in restricting tachyzoite to bradyzoite conversion through which the parasite evades the immune system and factors such as chemotherapeutic agents. Such inhibitors if developed would help combat acute on chronic infection in cases of reactivation if given prophylactically.

\subsubsection{Rhoptry kinases (ROP)}

The rhoptry kinases are secreted from rhoptry organelles situated at the neck of $T$. gondii. The ROPs contribute immensely in the pathogenesis of $T$. gondii from the initial stage of moving junction formation, gliding motility, the formation of the parasitophorous vacuole membrane (PVM), immune evasion as well as virulence [49]. The ROPs are highly divergent between the various strain types of the parasite while some are well conserved [50]. The diversity in these proteins is due to the differences in amino acid residues required for the catalytic activities. While some of the ROPs (ROP2, ROP4, ROP5, ROP7 and ROP8) lack the glycine loops and the conserved aspartic acid in the catalytic domain that is involved in phosphorylation activities, others such as ROP17, ROP16, and ROP18 were reported to have catalytic activities in vitro [51]. ROP2 families (ROP2, ROP4, ROP5, ROP7, ROP8), are the largest group of rophtry kinases associated with PVM upon secretion.
The ROP2 proteins coordinate a lot of activities that include cell invasion, replication, and the association between PVM and host cell organelles, immune evasion and virulence. These ROPs family were reported to share a common C-terminal transmembrane domain which is a PKs like domain and upstream of this PK region, they contain basic amino acids rich $\mathrm{N}$ terminal tail. The ROP2 bring host mitochondria in close association to the PVM. However, a study [52] showed that the recruitment of mitochondria to PVM is not necessarily a sole function of the ROP2 because a knockout study showed no defect in mitochondrial recruitment. ROP5 is a factor in virulence and this was proved by a targeted deletion that was followed by loss of virulence activity whereas restoration of which causes full blown pathogenesis in mice [51]. However, to compliment the activities of ROP18, ROP5 is expressed and this helps the ROP18 to inhibit the accumulation of the interferon gamma induced immunity related GTPases (IRGs) in a murine model. The function of the IRGs is to bind and disrupt the parasite enclosed within the PV under normal circumstances. This function is inhibited by the presence of ROP18, whose activity is enhanced by ROP5 [23] that leads to host cell immune evasion and hence pathogenesis. Moreover, some of the ROPs have no definite function but were reported to have conserved residue for kinase activity [23,51]. The ROPs targeted host cell nucleus was reported to have kinase domain catalytic ability to phosphorylate host cell molecules and hence modulating cell function and cell cycle [53]. The most studied among them are the ROP16 and RO18. While the former is expressed in type I and III genotypes, the latter is expressed in type I and type II and their overall function is to regulate host immune system thereby increasing the virulence of the parasite [54]. Regulation of the host immune system was reported to be due to the direct phosphorylation activity of ROP16 on STAT3 and STAT6 leading to down regulation of IL-12, a key cytokine in infection control by the immune system $[22,54]$.

It is also important to note that, the cytokine IFN$\mathrm{Y}$, that is expressed in fighting against $T$. gondii, excess of which is usually checked by IL-10 [54], can be controlled indirectly by ROP16. Direct phosphorylation of STAT3 can make $T$. gondii to mimic IL-10 and this can cause downregulation of the IFN-y thereby enhancing virulence in the host. ROP18, on the other hand, was reported to indirectly inhibit the activities of the IFN- $\mathrm{y}$ on immunity related GTPases (IRGs). The IRGs are 
activated by IFN-Y [55] which leads to the destruction of the PV. This action is inhibited by RPO18 which was reported to phosphorylate the IRGs thereby preserving the PV and continuous proliferation of the parasite by evading the immune system [22,54].

In search of a vaccine for toxoplasmosis, $T$. gondii PKs were reported to be promising candidates. Zhang et al. [34] reported that the combination of CDPK6 and ROP18 proteins in a vaccine were seen to confer immunity in mice subsequently infected with $\mathrm{RH}$ tachyzoite. The size of the brain cysts of the infected mice were reduced in comparison to the non-vaccinated control group. Prior to this study, the same group of mice immunized with pVAX-CDPK3 produced a significant increase in cytokines notably IFN- $\gamma$, IL-2, IL-12 and IL-23 [56]. The study further reported that the survival rates of the immunized mice were increased associated with reduction in brain cyst formation. For the essential role played by the CDPKs and ROP kinases in the pathogenesis of $T$. gondii, and because of their absence in mammalian host, drug and or vaccine development to these essential proteins is crucial at this moment to stop the spread of the parasite. The ROPs can also be used as therapeutic vaccines as proposed in

\section{APICOPLAST}

Apicoplast is an organelle that is present in apicomplexan protozoa with many metabolic activities. It is a non-photosynthetic organelle originated through endosymbiosis of green algae that is involved in biosynthetic activities of various metabolites and cofactors such as fatty acids, abscisic acid, lipoic acid, isoprenoids and heme [57]. The apicoplast is a good target for drug development owing to its characteristic nature and biosynthetic activities that resemble that of prokaryotes [7,21]. Many metabolic activities in this organelle are different from that of human as such it may be used as a drug target against important parasitic apicomplexan. The potential drug targets in apicoplast are discussed herein:

\subsection{Fatty Acid Metabolism}

Fatty acids are essential to every living cell because of the immense need for the synthesis of many intermediate products that are needed for many cellular processes. The life cycle of $T$. gondii demands for high supply of lipids to enable intracellular survival. This is evident by the formation of parasitophorous vacuole that houses the intracellular stage within the host cells. The vacuole is surrounded by three layered-membranes. Likewise, the essential organelles concentrated at the apical end of the parasite (micronemes, rophtry and dense granules) and the membrane-bound apicoplast [58].

The presence of type II fatty acid synthase II (FASII) in the apicoplast of $T$. gondii that is involved in fatty acid biosynthesis as found in prokaryotes, is essential for parasite survival and its absence in human, makes it a target for drug development [58]. Inhibition of enzymes that mediate important steps in type II fatty acid synthesis and its elongation in the apicoplast was shown to inhibit parasite growth $[59,60]$. More especially the acetyl CoA carboxylase (ACC) that catalyzes the formation of Malonyl-CoA, the $\beta$ ketoacyl ACP synthase III $(\mathrm{FabH})$, in the first condensation step and the enoyl ACP reductase (Fabl) that catalyzes the final step, are very important drug targets. Several compounds such as clodinafop, thiolactomycin and triclosan (Table 1) were shown to have inhibitory activities on these enzymes and block fatty acid synthesis that results to the death of parasite [61]. And, their absence in human, targeting these enzymes in $T$. gondii will prove beneficial in combating toxoplasmosis.

Table 1. Potential therapeutic targets in apicoplast

\begin{tabular}{lllll}
\hline Enzymes & Inhibitor & Organism & $\mathbf{I C}_{50}$ & Reference \\
\hline Enoyl-ACP reductase & Triclosan & T.gondii & $62 \mathrm{ng} / \mathrm{mL}$ & {$[65]$} \\
& & P. falciparum & $<2000 \mathrm{ng} / \mathrm{mL}$ & \\
B-Ketoacyl-ACP $(\mathrm{FabH})$ & Thiolactomycin & P. falciparum & $50 \mu \mathrm{M}$ & {$[66]$} \\
AcetylCoA carboxylase & Clodinafop & T. gondii & $10-100 \mu \mathrm{M}$ & {$[67]$} \\
DXP reductoisomerase & Fosmidomycin & P. falciparum & $350 \mathrm{nM}$ & {$[62]$} \\
& & T. gondii & $>500 \mathrm{nM}$ & {$[63,64]$} \\
DNA gyrase & \multirow{2}{*}{ Ciprofloxacin } & T. gondii & $30 \mu \mathrm{M}$ & {$[68]$} \\
& & P. falciparum & $50 \mu \mathrm{M}$ & {$[69]$} \\
& & C. parvum & $80 \mu \mathrm{g} / \mathrm{mL}$ & {$[70]$} \\
\hline
\end{tabular}

$I C_{50}: 50 \%$ inhibitory concentration 


\subsection{Isoprenoid Synthesis}

Isoprenoid is important in cell signaling for the control of many cellular activities, protein prenylation, and as a precursor of sterols and ubiquinone. A non-mevalonate pathway, 1deoxy-D-xylulose-5-phosphate (DOXP) was reported to be present in the apicoplast. The DOXP, was reported [62] to be responsible for isoprenoid biosynthesis in $P$. falciparum. The pathway differs from that obtained in the cytosol of other eukaryotes in the initial substrate for the synthesis of isoprenoid precursors; isopentenyl pyrophosphate (IPP) and dimethylallyl diphosphate (DMAPP). The IPP and DMAPP are formed through condensation of glyceraldehyde 3-phosphate and pyruvate in DOXP pathway while in mevalonate pathway in eukaryotes and archaebacteria, the synthesis of isoprenoids is through condensation of acetyl CoA and acetoacetyl CoA to form 3-hydroxy-3methylglutaryl-CoA (HMG-CoA). Interestingly, the two key enzymes featured in DOXP pathway i.e DXP reductoisomerase and DXP synthase found in $P$. falciparum [62] were also reported to be present in $T$. gondii and very essential for the parasite's survival. The absence of these two enzymes in human makes them good drug targets [61]. Fosmidomycin antibiotic that inhibits the activities of DXP reductoisomerase and DXP synthase was reported to inhibit the replication of Plasmodium vinckei in mice and $P$. falciparum [62]. Since $T$. gondii and related apicomplexan share the same enzymes in the DOXP pathway, sensitivity to fosmidomycin is anticipated. But the activity of this antibiotic on $T$. gondii was resisted $[63,64]$ except at higher concentrations (Table 1). That is attributed to a decrease uptake of the drugs by the infected cells [61], the nature of the cell and possible efflux of the antibiotic from the parasite [64] hence significantly affecting its bioavailability. Therefore, at a higher concentration, the fosmidomycin antibiotic can suppress the growth of $T$. gondii.

\subsection{DNA Replication}

DNA replication in the apicoplast of $T$. gondii follows sequential order as seen in prokaryotes. The apicoplast contains a circular DNA which is $35-\mathrm{kb}$ in size and therefore unwinds before the replication process as in bacteria. The sequence of events regarding the unwinding of the circular DNA before replication and its subsequent supercoiling are mediated by DNA topoisomerase [71]. DNA gyrase found in bacteria, [72] is a type II DNA topoisomerase that is also present in apicomplexan. DNA gyrase is responsible for DNA replication in apicoplast $[71,73]$. Several quinolone antibiotics are reported to inhibit DNA gyrase and that affect parasite growth as observed in bacteria. A notable member of such class, ciprofloxacin has been reported by studies to inhibit the growth of $P$. falciparum [69], T. gondii [68] and Cryptosporidum parvum [70]. It was also reported by McFadden and Roos [74] that a fluoroquinolone, trovafloxacin inhibit $T$. gondii growth in vitro at a dose range of $0.77-0.98$ $\mu \mathrm{g} / \mathrm{mL}$. Ciprofloxacin, ofloxacin and temafloxacin were shown additionally effective. The finding from the in vivo study indicated significant parasite clearance in a dose-dependent manner. The quinolones are reported to inhibit DNA gyrase, hence inhibiting proper DNA replication which results in the death of the parasite [74]. Since DNA gyrase is of prokaryotic origin, and hence absent in human, design and synthesis of an inhibitor, potentially not so toxic to human, can be arrived at to bypass the possibility of resistance as seen with ciprofloxacin in bacteria [75].

\subsection{Protein Synthesis}

Protein synthesis in the apicoplast occurs independently from that of the parasite's nucleus. This is because the apicoplast genome encodes only for components of gene expression and protein synthesis [76]. It encodes for a complete set of enzymes and RNAs that are indispensable for ribosome assembly. Like in Plasmodium parasite, these set of enzymes and RNAs include the rRNA (small and large subunit), all tRNAs, and translation elongation factor -Tu $[75,77]$. It is important to note that, antibiotics used for the treatment of toxoplasmosis such as clindamycin, azithromycin and spiramycin were proven to have activities on protein synthesis in prokaryotic organisms by binding to ribosomal subunits. These antibiotics were reported to have in vitro and in vivo anti-parasitic activities. The study by Pfefferkorn et al [78] reported that clindamycin has parasiticidal activity on $T$. gondii at a very low concentration despite its delayed onset of action and that this effect increases with increase in concentration. Though the study did not investigate the mechanism through which it caused its effect, the authors suggest that the antibiotic might have had the same mechanism of action like in bacteria by interfering with protein synthesis. Since the protein synthesis in apicoplast is of prokaryotic type, all its components may be suitable for specific drug 
development against the parasite and other related apicomplexans.

\subsection{Heme Metabolism}

Heme is an essential cofactor of several enzymes such as the catalase and the cytochromes. Heme biosynthesis occurs in $T$. gondii as in other apicomplexans, prokaryotes, plants and animals. But a striking difference is that in $T$. gondii, this activity is split between the apicoplast, the mitochondrion and the cytosol [61]. This depends on the location of enzymes in the biosynthetic pathways. In those organisms that are equipped with machineries for the de novo heme biosynthesis such as Plasmodium spp, T. gondii, fungi, plants and animals [79,80], the initial committed precursor, aminolevulinic acid (ALA) is the same but synthesized through different substrate utilization [79]. The ALA is the first precursor of heme, synthesized in the mitochondrion from glycine and succinyl-CoA which must go through different enzymatic steps in both photosynthetic and non-photosynthetic eukaryotes. The first catalytic conversion of ALA to porphobilinogen is mediated by porphobilinogen synthase (PBGS). However, because the PBGS is of plant origin makes it a good drug target against $T$. gondii [78] and its inhibition can lead to the death of the parasite.

\section{HEAT SHOCK PROTEINS (HSPs)}

Heat shock proteins (HSPs) are well conserved in microorganisms and contribute in host cell invasion, growth, survival and stage conversion from tachyzoite to bradyzoite stage in $T$. gondii infection [81]. During T. gondii infection, HSPs (Hsp100, Hsp90, Hsp70, Hsp60, Hsp40) expression is induced by stress factors that favor formation of bradyzoite. The HSPs play an important role in maintaining protein homeostasis which enhances intracellular survival of $T$. gondii [82]. A gene knockout study [81] reported that the Hsp90 knockout parasites were unable to establish infection in Vero cells and that the parasites had a short life span in mice. This indicates that the absence of this protein seriously affects the ability of the parasite to invade the Vero cells and cannot run a chronic bradyzoite stage in vivo and hence has decreased virulence. Hsp70 and Hsp60 are nuclear encoded proteins and their role in the post-translational assembly of several proteins make them a good target for drug development. A study [83] reported that Hsp60 is responsible for stage specific induction of respiratory pathway whereas $\mathrm{Hsp} 70$ is involved in stage differentiation and virulence. As part of its virulence feature, Hsp70 was reported to protect the parasite from the immune system of the host. Dobbin et al. [84] conducted a study in which quercetin and oligonucleotide were used to treat virulent (RH, ENT) and avirulent (ME49) T. gondii strains. The study revealed a reduction in expression of Hsp70 in virulent strain and their proliferation was reduced in mice. However, the result from the in vitro study showed that the tachyzoite rapidly converts to bradyzoite. The study further reported that the inducible nitric oxide synthase (iNOS) was increased in RAW 264.7 cells after infection with Hsp70 reducedvirulent parasite. This finding confirmed that the Hsp70 protein altered production of Nitric oxide (NO) which takes part in immune control of toxoplasmosis. Therefore, any drug that can inhibit the activity of Hsp70 will prevent immune evasion by the parasite.

\section{METABOLIC ENZYMES OF T. gondii}

Study on the similarities between the human host and $T$. gondii with respect to their metabolic pathways was reported by Gautam et al. [6]. According to the study, 11 potential enzymes that can be used as drug targets were identified in $T$. gondii which were absent in human. Some of the enzymes appear to play a significant role in more than one pathway. Among the 11 enzymes, is glutamate dehydrogenase enzyme (GLDH) which appears to catalyse many metabolic reactions such as amino acid metabolism and oxidation reaction. GLDH catalyses the formation of NADPH which is needed to reduce the oxidised forms of glutathione and thioredoxin in the presence of glutathione reductase (GR) and thioredoxin reductase (TrxR) respectively. The GLDH is unique only in $T$. gondii (and plasmodium) as such it is suitable to be a drug target as in Plasmodium parasite [85]. Other important enzymes in glucose, amino acid and purine metabolisms were identified to be essential in the parasite but absent in human. Metabolic differences between the various stages of the parasite can serve as a drug target. Differences in metabolism exist between the actively dividing tachyzoite and the slowly replicating bradyzoite [86]. In both stages of the infection, lactate is produced as a source of energy in the glycolytic pathway but differ in types of lactate dehydrogenase (LDH) activity. The two isoforms LDH1 in tachyzoite and LDH2 in bradyzoite catalyze the conversion of pyruvate to lactate in anaerobic condition. The activity of 
LDH is reported to be higher in bradyzoite [87]. In previous study [88], the LDH genes knockdown $T$. gondii were shown to have impaired growth in vitro. The study further showed that, the tachyzoite stage of the parasite was unable to establish chronic infection in vivo. Furthermore, the mice infected with LDH knockdown tachyzoite were observed to tolerate subsequent infection when challenged with a maximum infective dose of $10^{4}$ tachyzoites. This study shows that developing a live LDH attenuated tachyzoites can be used as a potential vaccine candidate against toxoplasmosis. This finding was recently supported by a study [89] which stressed that LDH is involved in virulence and bradyzoite differentiation of the parasite and that LDH mutant parasite can be used for vaccine development. Glucose - phosphate-isomerase (GPI) present in the bradyzoite is important in glucose metabolism and appears to be a good target because it differs from that of the humans [21]. Another important enzyme in the parasite purine pathway, adenosine kinase, was also reported to be inhibited by analogues of 7-deaza6-benzylthioinosine with significant anti parasitic activities [90].

Blume et al. [91] reported another important gluconeogenic enzyme, fructose 1,6bisphosphatase, which exists in two isoforms; TgFBP1 and TgFBP2. Although, the enzyme shares properties with that of prokaryotes, unlike the prokaryotes and other eukaryotes that express this enzyme in glucose depletion state, the enzyme is constitutively expressed in $T$. gondii. The two isoforms are essential in either glucose depleted or repleted situations and they are required for replication in vitro and for virulence in vivo [91]. This finding was a result of a conditional knockdown of TgFBP2 that causes reduction in the activities of the enzyme which resulted in the loss of intracellular replication and acute virulence in mice. With this, it was concluded that this enzyme could be a potential drug target.

For intracellular replication and membrane biogenesis, $T$. gondii acquires cholesterol from host cell through a low-density lipoprotein pathway (LDLP) and convert it to cholesterol esters (CE) that are stored in lipid bodies [92]. In fact, a typical organelle with a very high membrane cholesterol level is rhoptry which secretes proteins that facilitates cell invasion [58]. Previous report [93] showed that statins have an anti-proliferative effect on $T$. gondii. The ability of this parasite to convert host cell derived cholesterol and other lipids to CE and stored in lipid bodies is achieved due to activities of certain enzymes from the endoplasmic reticulum. These enzymes are acyl-CoA: cholesterol acyltranferase (ACAT: ACAT1 \& ACAT2) whose susceptibility to inhibitors ware found to be different from that of mammals [57]. In addition, the most abundant phospholipids in $T$. gondii is phosphatidylcholine [94]. The parasite takes choline, serine and ethanolamine from the surrounding, as precursors for other phospholipids. However, interference with choline metabolism has been shown to inhibit the growth of $T$. gondii [94] and P. falciparum [95]. Although it has not been observed or reported in humans, patients on statins undergoing treatment for hyperlipidemia, the activity of statins may retard $T$. gondii infection progression in human host and thus, inhibition of choline uptake appears to be a good target for drug discovery. Enoyl reductase involved in type II fatty acid biosynthesis was also identified to be inhibited by some compounds [96] and most importantly the enzyme is lacking in animals. Since triclosan was successfully used to inhibit enoyl reductase [97], a derivative of this compound with low toxicity can be used for the treatment of $T$. gondii infection.

\section{CONCLUSION}

Treatment of intracellular pathogens can be so challenging because the active ingredient may have to penetrate tissue layers to reach its targets. T. gondii is one of those intracellular parasites that survive only within the cell in a confined non-fusogenic vacuole that prevents the action of lysozymes against the parasite. In addition, because of the characteristic features of the different stages of its life cycle, $T$. gondii is potentially difficult to eliminate. The infective stages of the parasite can be targeted at a different site if only their physiological characteristic and biological interaction with host agents are well studied. Therefore, drug development should take into consideration, the essentiality and specificity of the targets, to solve the problems of adverse drug reaction and clearance of the parasite at each stage.

\section{ACKNOWLEDGMENT}

Malaysia Ministry of Education through Fundamental Research Grant Scheme [grant number FRGS/1/2015/SKK12/UPM/03/01] 


\section{COMPETING INTERESTS}

Authors have declared that no competing interests exist.

\section{REFERENCES}

1. Montazeri M, Sharif M, Sarvi S, Mehrzadi S, Ahmadpour E, Daryani A. A systematic review of in vitro and in vivo activities of anti-toxoplasma drugs and compounds (2006-2016). Frontiers in Microbiology. 2017;8

2. Yan C, Liang LJ, Zheng $K Y$, Zhu XQ. Impact of environmental factors on the emergence, transmission and distribution of Toxoplasma gondii. Parasites and vectors. 2016;9(1):137.

3. Doliwa C, Escotte-Binet S, Aubert D, Sauvage V, Velard F, Schmid A, Villena I. Sulfadiazine resistance in Toxoplasma gondii: No involvement of over expression or polymorphisms in genes of therapeutic targets and ABC transporters. Parasite. 2013;20:19.

4. Hunter CA, Sibley LD. Modulation of innate immunity by Toxoplasma gondii virulence effectors. Nature Reviews Microbiology. 2012;10(11):766.

5. Alday PH, Doggett JS. Drugs in development for toxoplasmosis: Advances, challenges, and current status. Drug Design, Development and Therapy. 2017; 11:273.

6. Gautam B, Singh G, Wadhwa G, Farmer $R$, Singh $S$, Singh AK, Yadav PK. Metabolic pathway analysis and molecular docking analysis for identification of putative drug targets in Toxoplasma gondii: novel approach. Bioinformation. 2012;8(3): 134-141.

7. Antczak M, Dzitko $\mathrm{K}$, Długońska $\mathrm{H}$. Human toxoplasmosis-Searching for novel chemotherapeutics. Biomedicine \& Pharmacotherapy. 2016;82:677-684.

8. McAuley JB. Congenital toxoplasmosis. Journal of the Pediatric Infectious Diseases Society. 2014;3(Suppl_1):S30S35.

9. Julliac B, Theophile H, Begorre M, Richez $B$, Haramburu F. Side effects of spiramycin masquerading as local anesthetic toxicity during labor epidural analgesia. International Journal of Obstetric Anesthesia. 2010;19(3):331-332.

10. Habib FA. Post-treatment assessment of acute Toxoplasma infection during pregnancy. Journal of Obstetrics and Gynaecology. 2008;28(6):593-595.

11. Paquet $\mathrm{C}$, Yudin $\mathrm{MH}$, Allen VM, Bouchard C, Boucher M, Caddy S, Van Schalkwyk J. Toxoplasmosis in pregnancy: Prevention, screening, and treatment. Journal of Obstetrics and Gynaecology Canada. 2013;35(1):78-79.

12. Bodaghi B, Touitou V, Fardeau C, Paris L, LeHoang P. Toxoplasmosis: New challenges for an old disease. Eye. 2012; 26(2):241.

13. Montoya JG, Liesenfeld O. Toxoplasmosis. Lancet. 2004;363:1965-1976.

14. Alves CF, Vitor RWA. Efficacy of atovaquone and sulfadiazine in the treatment of mice infected with Toxoplasma gondii strains isolated in Brazil. Parasite. 2005;12(2):171-177.

15. Sims PF. Drug resistance in Toxoplasma gondii. In Antimicrobial Drug Resistance Humana Press. 2009;1121-1126.

16. Aspinall TV, Joynson DH, Guy E, Hyde JE, Sims PF. The molecular basis of sulfonamide resistance in Toxoplasma gondii and implications for the clinical management of toxoplasmosis. Journal of Infectious Diseases. 2002;185(11):16371643.

17. Seeber F, Steinfelder S. Recent advances in understanding apicomplexan parasites. F1000Research. 2016;5.

18. McFadden DC, Camps M, Boothroyd JC. Resistance as a tool in the study of old and new drug targets in Toxoplasma. Drug Resistance Updates. 2001;4(2):79-84.

19. McFadden DC, Tomavo S, Berry EA, Boothroyd JC. Characterization of cytochrome b from Toxoplasma gondii and Qo domain mutations as a mechanism of atovaquone-resistance. Molecular and Biochemical Parasitology. 2000;108(1):112.

20. Meneceur P, Bouldouyre MA, Aubert D, Villena I, Menotti J, Sauvage V, Derouin F. In vitro susceptibility of various genotypic strains of Toxoplasma gondii to pyrimethamine, sulfadiazine, and atovaquone. Antimicrobial Agents and Chemotherapy. 2008;52(4):1269-1277.

21. Müller J, Hemphill A. New approaches for the identification of drug targets in protozoan parasites. In International Review of Cell and Molecular Biology. Academic Press. 2013;301:359-401.

22. Lim DC, Cooke BM, Doerig C, Saeij JP. Toxoplasma and Plasmodium protein 
kinases: Roles in invasion and host cell remodeling. International Journal for Parasitology. 2012;42(1):21-32.

23. Wei F, Wang W, Liu Q. Protein kinases of Toxoplasma gondii: Functions and drug targets. Parasitology Research. 2013; 112(6):2121-2129.

24. Wang $\mathrm{Y}$, Yin $\mathrm{H}$. Research advances in microneme protein 3 of Toxoplasma gondii. Parasites \& Vectors. 2015;8(1):384.

25. Peixoto L, Chen F, Harb OS, Davis PH, Beiting DP, Brownback CS, Roos DS. Integrative genomic approaches highlight a family of parasite-specific kinases that regulate host responses. Cell Host \& Microbe. 2010;8(2):208-218.

26. Ojo KK, Larson ET, Keyloun KR, Castaneda LJ, DeRocher AE, Inampudi KK, Napuli AJ. Toxoplasma gondii calciumdependent protein kinase 1 is a target for selective kinase inhibitors. Nature Structural and Molecular Biology. 2010; 17(5):602.

27. Kato K, Sugi T, Iwanaga T. Roles of apicomplexan protein kinases at each life cycle stage. Parasitology International. 2012;61(2):224-234.

28. Morlon-Guyot J, Berry L, Chen CT, Gubbels MJ, Lebrun M, Daher W. The Toxoplasma gondii calcium-dependent protein kinase 7 is involved in early steps of parasite division and is crucial for parasite survival. Cellular Microbiology. 2014;16(1):95-114.

29. Doggett JS, Ojo KK, Fan E, Maly DJ, Van Voorhis WC. Bumped kinase inhibitor 1294 treats established Toxoplasma gondii infection. Antimicrobial agents and Chemotherapy. 2014;58(6):3547-3549.

30. Moine E, Dimier-Poisson I, EnguehardGueiffier C, Logé C, Pénichon M, Moiré N, Gueiffier A. Development of new highly potent imidazo [1, 2-b] pyridazines targeting Toxoplasma gondii calciumdependent protein kinase 1. European Journal of Medicinal Chemistry. 2015;105: 80-105.

31. Zhang Z, Ojo KK, Johnson SM, Larson ET, He P, Geiger JA, Maly DJ. Benzoylbenzimidazole-based selective inhibitors targeting Cryptosporidium parvum and Toxoplasma gondii calcium-dependent protein kinase-1. Bioorganic \& Medicinal Chemistry Letters. 2012;22(16):5264-5267.

32. Lourido S, Shuman J, Zhang C, Shokat KM, Hui R, Sibley LD. Calcium-dependent protein kinase 1 is an essential regulator of exocytosis in Toxoplasma. Nature. 2010; 465(7296):359.

33. Chen K, Wang JL, Huang SY, Yang WB, Zhu WN, Zhu XQ. Immune responses and protection after DNA vaccination against Toxoplasma gondii calcium-dependent protein kinase 2 (TgCDPK2). Parasite. 2017;24:41.

34. Zhang NZ, Xu Y, Wang M, Chen J, Huang SY, Gao Q, Zhu XQ. Vaccination with Toxoplasma gondii calcium-dependent protein kinase 6 and rhoptry protein 18 encapsulated in poly (lactide-co-glycolide) microspheres induces long-term protective immunity in mice. BMC Infectious Diseases. 2016;16(1):168.

35. McCoy JM, Whitehead L, van Dooren GG, Tonkin CJ. TgCDPK3 regulates calciumdependent egress of Toxoplasma gondii from host cells. PLoS Pathogens. 2012; 8(12):e1003066.

36. Garrison E, Treeck M, Ehret E, Butz H, Garbuz T, Oswald BP, Arrizabalaga G. A forward genetic screen reveals that calcium-dependent protein kinase 3 regulates egress in Toxoplasma. PLoS Pathogens. 2012;8(11):e1003049.

37. Dan-Goor M, Nasereddin A, Jaber H, Jaffe CL. Identification of a secreted casein kinase 1 in Leishmania donovani: Effect of protein over expression on parasite growth and virulence. PLoS One. 2013;8(11): e79287.

38. Allocco JJ, Donald R, Zhong T, Lee A, Tang YS, Hendrickson RC, Nare B. Inhibitors of casein kinase 1 block the growth of Leishmania major promastigotes in vitro. International Journal for Parasitology. 2006;36(12):1249-1259.

39. Donald RG, Zhong T, Meijer L, Liberator PA. Characterization of two T. gondii CK1 isoforms. Molecular and Biochemical Parasitology. 2005;141(1):15-27.

40. Wei S, Marches F, Daniel B, Sonda S, Heidenreich K, Curiel T. Pyridinylimidazole p38 mitogen-activated protein kinase inhibitors block intracellular Toxoplasma gondii replication. International Journal for Parasitology. 2002;32(8):969-977.

41. Brumlik MJ, Wei S, Finstad K, Nesbit J, Hyman LE, Lacey M, Curiel TJ. Identification of a novel mitogen-activated protein kinase in Toxoplasma gondii. International Journal for Parasitology. 2004;34(11): 1245-1254.

42. Brumlik MJ, Pandeswara S, Ludwig SM, Jeansonne DP, Lacey MR, Murthy K, 
Hurez V. TgMAPK1 is a Toxoplasma gondii MAP kinase that hijacks host MKK3 signals to regulate virulence and interferon- $\gamma$-mediated nitric oxide production. Experimental Parasitology. 2013;134(3): 389-399.

43. Huang $\mathrm{H}$, Fen $\mathrm{Y}$, Bao $\mathrm{Y}$, Lee $\mathrm{H}$, Lisanti MP, Tanowitz H, Weiss LM. Molecular cloning and characterization of mitogenactivated protein kinase 2 in Toxoplasma gondii. Cell Cycle. 2011;10(20):3519-3526.

44. Donald RG, Allocco J, Singh SB, Nare B, Salowe SP, Wiltsie J, Liberator PA. Toxoplasma gondii cyclic GMP-dependent kinase: Chemotherapeutic targeting of an essential parasite protein kinase. Eukaryotic Cell. 2002;1(3):317-328.

45. Gurnett AM, Liberator PA, Dulski PM, Salowe SP, Donald RG, Anderson JW, Darkin-Rattray SJ. Purification and molecular characterization of cGMP-dependent protein kinase from apicomplexan parasites a novel chemotherapeutic target. Journal of Biological Chemistry. 2002;277(18): 15913-15922.

46. Cardew EM, Verlinde CL, Pohl E. The calcium-dependent protein kinase 1 from Toxoplasma gondii as target for structurebased drug design. Parasitology. 2018; 145(2):210-218.

47. Kurokawa $H$, Kato $K$, Iwanaga $T$, Sugi $T$, Sudo A, Kobayashi K, Akashi H. Identification of Toxoplasma gondii cAMP dependent protein kinase and its role in the tachyzoite growth. PLoS One. 2011;6(7):e22492.

48. Sugi T, Ma YF, Tomita T, Murakoshi F, Eaton MS, Yakubu R, Gupta N. Toxoplasma gondii cyclic AMP-dependent protein kinase subunit 3 is involved in the switch from tachyzoite to bradyzoite development. MBio. 2016;7(3):e0075516.

49. Niedelman W, Gold DA, Rosowski EE, Sprokholt JK, Lim D, Arenas AF, Saeij JP. The rhoptry proteins ROP18 and ROP5 mediate Toxoplasma gondii evasion of the murine, but not the human, interferongamma response. PLoS Pathogens 2012; 8(6):e1002784.

50. Sibley LD. Development of forward genetics in Toxoplasma gondii. International Journal for Parasitology. 2009; 39(8):915-924.

51. El Hajj H, Demey E, Poncet J, Lebrun M, Wu B, Galéotti N, Dubremetz JF. The ROP2 family of Toxoplasma gondii rhoptry proteins: Proteomic and genomic charac- terization and molecular modeling. Proteomics. 2006;6(21):5773-5784.

52. Pernas L, Boothroyd JC. Association of host mitochondria with the parasitophorous vacuole during Toxoplasma infection is not dependent on rhoptry proteins ROP2/8. International Journal for Parasitology. 2010;40(12):1367-1371.

53. Behnke MS, Khan A, Wootton JC, Dubey JP, Tang K, Sibley LD. Virulence differences in Toxoplasma mediated by amplification of a family of polymorphic pseudokinases. Proceedings of the National Academy of Sciences. 2011; 108(23):9631-9636.

54. Blader IJ, Saeij JP. Communication between Toxoplasma gondii and its host: Impact on parasite growth, development, immune evasion and virulence. APMIS. 2010;117(5-6):458-476.

55. Felix Y. Innate immunity to Toxoplasma gondii infection. Nature Reviews Immunology. 2014;14:109-121.

56. Zhang NZ, Huang SY, Zhou DH, Chen J, $\mathrm{Xu} \mathrm{Y,} \mathrm{Tian} \mathrm{WP,} \mathrm{Zhu} \mathrm{XQ.} \mathrm{Protective}$ immunity against Toxoplasma gondii induced by DNA immunization with the gene encoding a novel vaccine candidate: Calcium-dependent protein kinase 3. BMC Infectious Diseases. 2013;13(1):512.

57. Saremy S, Boroujeni ME, Bhattacharjee B, Mittal V, Chatterjee J. Identification of potential apicoplast associated therapeutic targets in human and animal pathogen Toxoplasma gondii ME49. Bioinformation. 2011;7(8):379-83.

58. Sonda S, Hehl AB. Lipid biology of Apicomplexa: Perspectives for new drug targets, particularly for Toxoplasma gondii. TRENDS in Parasitology. 2006;22(1):4147.

59. Gornicki P. Apicoplast fatty acid biosynthesis as a target for medical intervention in apicomplexan parasites. International Journal for Parasitology. 2003;33(9):885896.

60. Seeber F. Biosynthetic pathways of plastid-derived organelles as potential drug targets against parasitic apicomplexa. Current Drug Targets-Immune, Endocrine \& Metabolic Disorders. 2003;3(2):99-109.

61. Seeber F, Soldati-Favre D. Metabolic pathways in the apicoplast of apicomplexa. International Review of Cell and Molecular Biology. 2010;281:161-228.

62. Jomaa H, Wiesner J, Sanderbrand S, Altincicek $B$, Weidemeyer $C$, Hintz $M$, 
Türbachova I, Eberl M, Zeidler J, Lichtenthaler HK. Inhibitors of the nonmevalonate pathway of isoprenoid biosynthesis as antimalarial drugs. Science. 1999;285:1573-1576.

63. Ling Y, Sahota G, Odeh S, Chan JM, Araujo FG, Moreno SN, Oldfield E. Bisphosphonate inhibitors of Toxoplasma gondii growth: In vitro, QSAR, and in vivo investigations. Journal of Medicinal Chemistry. 2005;48(9):3130-3140.

64. Clastre M, Goubard A, Prel A, Mincheva Z, Viaud-Massuart MC, Bout D, Laurent F. The methylerythritol phosphate pathway for isoprenoid biosynthesis in coccidia: Presence and sensitivity to fosmidomycin. Experimental Parasitology. 2007;116(4): 375-384.

65. McLeod R, Muench SP, Rafferty JB, Kyle DE, Mui EJ, Kirisits MJ, Dorris M. Triclosan inhibits the growth of Plasmodium falciparum and Toxoplasma gondii by inhibition of Apicomplexan Fab I. International Journal for Parasitology. 2001;31(2):109-113.

66. Waller RF, Keeling PJ, Donald RG, Striepen B, Handman E, Lang-Unnasch N, McFadden GI. Nuclear-encoded proteins target to the plastid in Toxoplasma gondii and Plasmodium falciparum. Proceedings of the National Academy of Sciences. 1998;95(21):12352-12357.

67. Zuther E, Johnson JJ, Haselkorn R, McLeod R, Gornicki P. Growth of Toxoplasma gondii is inhibited by aryloxyphenoxypropionate herbicides targeting acetyl-CoA carboxylase. Proceedings of the National Academy of Sciences. 1999; 96(23):13387-13392.

68. Gozalbes R, Brun-Pascaud M, GarciaDomenech R, Galvez J, Girard PM, Doucet JP, Derouin F. Anti-toxoplasma activities of 24 quinolones and fluoroquinolones in vitro: Prediction of activity by molecular topology and virtual computational techniques. Antimicrobial Agents and Chemo-therapy. 2000;44(10):27712776.

69. Divo AA, Sartorelli AC, Patton CL, Bia FJ. Activity of fluoroquinolone antibiotics against Plasmodium falciparum in vitro. Antimicrobial Agents and Chemotherapy. 1988;32(8):1182-1186.

70. Woods KM, Nesterenko MV, Upton SJ. Efficacy of 101 antimicrobials and other agents on the development of Cryptosporidium parvum in vitro. Annals of
Tropical Medicine \& Parasitology. 1996; 90(6):603-615.

71. García-Estrada C, Prada CF, FernándezRubio C, Rojo-Vázquez $F$, Balaña-Fouce R. DNA topoisomerases in apicomplexan parasites: Promising targets for drug discovery. Proceedings of the Royal Society of London B: Biological Sciences. 2010;277(1689):1777-1787.

72. Maxwell A. DNA gyrase as a drug target. Trends in Microbiology. 1997;5(3):102-109.

73. McFadden GI, Roos DS. Apicomplexan plastids as drug targets. Trends in Microbiology. 1999;7(8):328-333.

74. Khan AA, Slifer T, Araujo FG, Remington JS. Trovafloxacin is active against Toxoplasma gondii. Antimicrobial agents and Chemotherapy. 1996;40(8):1855-1859.

75. Ralph SA, D'Ombrain MC, McFadden GI. The apicoplast as an antimalarial drug target. Drug Resistance Updates. 2001; 4(3):145-151.

76. Reiff SB, Vaishnava S, Striepen B. The $\mathrm{HU}$ protein is important for apicoplast genome maintenance and inheritance in Toxoplasma gondii. Eukaryotic Cell. 2012; 11(7):905-915.

77. Lüder CG, Seeber F. Toxoplasma. In molecular parasitology. Springer, Vienna. 2016;217-239.

78. Pfefferkorn ER, Nothnagel RF, Borotz SE. Parasiticidal effect of clindamycin on Toxoplasma gondii grown in cultured cells and selection of a drug-resistant mutant. Antimicrobial Agents and Chemotherapy. 1992;36(5):1091-1096.

79. Shanmugam D, Wu B, Ramirez $U$, Jaffe EK, Roos DS. Plastid-associated Porphobilinogen Synthase from Toxoplasma gondii kinetic and structural properties validate therapeutic potential. Journal of Biological Chemistry. 2010; 285(29):22122-22131.

80. van Dooren GG, Kennedy AT, McFadden GI. The use and abuse of heme in apicomplexan parasites. Antioxidants \& Redox Signaling. 2012;17(4):634-656.

81. Sun H, Zhuo X, Zhao X, Yang Y, Chen X, Yao C, Du A. The heat shock protein 90 of Toxoplasma gondii is essential for invasion of host cells and tachyzoite growth. Parasite. 2017;24.

82. Ashwinder K, Kho MT, Chee PM, Lim WZ, Yap IK, Choi SB, Yam WK. Targeting heat shock proteins 60 and 70 of Toxoplasma gondii as a potential drug target: In silico approach. Interdisciplinary Sciences: 
Computational Life Sciences. 2016;8(4): 374-387.

83. Toursel C, Dzierszinski $F$, Bernigaud A, Mortuaire M, Tomavo S. Molecular cloning, organellar targeting and developmental expression of mitochondrial chaperone HSP60 in Toxoplasma gondii. Molecular and Biochemical Parasitology. 2000;111(2): 319-332.

84. Dobbin CA, Smith NC, Johnson AM. Heat shock protein 70 is a potential virulence factor in murine Toxoplasma infection via immunomodulation of host NF-KB and nitric oxide. The Journal of Immunology. 2002;169(2):958-965.

85. Müller S, Liebau E, Walter RD, KrauthSiegel RL. Thiol-based redox metabolism of protozoan parasites. Trends in Parasitology. 2003;19(7):320-328.

86. Weiss LM, Kim K. The development and biology of bradyzoites of Toxoplasma gondii. Front Biosci. 2011;5:D391-D405.

87. Denton H, Robert CW, Alexander J, Thong $\mathrm{KW}$, Coombs $\mathrm{GH}$. Enzymes of energy metabolism in the bradyzoite and tachyzoites of Toxoplasma gondii. FEMS Microbio Lett. 1996;137:103-8.

88. Al-Anouti F, Tomavo S, Parmley S, Ananvoranich $\mathrm{S}$. The expression of lactate dehydrogenase is important for the cell cycle of Toxoplasma gondii. Journal of Biological Chemistry. 2004;279(50):5230052311.

89. Abdelbaset AE, Fox BA, Karram MH, Ellah MRA, Bzik DJ, Igarashi M. Lactate dehydrogenase in Toxoplasma gondii controls virulence, bradyzoite differentiation and chronic infection. PloS One. 2017; 12(3):e0173745.

90. Kim YA, Sharon A, Chu CK, Rais RH, Al Safarjalani ON, Naguib FN, el Kouni MH. Structure- activity relationships of 7deaza-6-benzylthioinosine analogues as ligands of Toxoplasma gondii adenosine kinase. Journal of Medicinal Chemistry. 2008;51(13):3934-3945.
91. Blume M, Nitzsche R, Sternberg U, Gerlic M, Masters SL, Gupta N, McConville MJ. A Toxoplasma gondii gluconeogenic enzyme contributes to robust central carbon metabolism and is essential for replication and virulence. Cell Host \& Microbe. 2015; 18(2):210-220.

92. Coppens I, Sinai AP, Joiner KA. Toxoplasma gondii exploits host lowdensity lipoprotein receptor-mediated en ocytosis for cholesterol acquisition. J Cell Bio. 2000;149:167-180.

93. Sanfelice RA, da Silva SS, Bosqui LR, Miranda-Sapla MM, Barbosa BF, Silva RJ, Conchon-Costa I. Pravastatin and simvastatin inhibit the adhesion, replication and proliferation of Toxoplasma gondii ( $\mathrm{RH}$ strain) in HeLa cells. Acta Tropica. 2017; 167:208-215.

94. Gupta Nishith, Matthew M. Zahn, Isabelle Coppens, Keith A. Joiner, Dennis R. Voelker. Selective disruption of phosphatidylcholine metabolism of the intracellular parasite Toxoplasma gondii arrests its growth. The Journal of Biological Chemistry. 2005;280(16): 16345-16353.

95. Ancelin ML, Vial HJ. Quartenary ammonium compounds efficiently inhibit Plasmodium falciparum growth in vitro by impairment of choline transport. Antimicrobial Agents and Chemotherapy. 1986;29(5):814-820.

96. Tipparaju SK, Muench SP, Mui EJ, Ruzheinikov SN, Lu JZ, Hutson SL, Kozikowski AP. Identification and development of novel inhibitors of Toxoplasma gondii enoyl reductase. Journal of Medicinal Chemistry. 2010;53(17):62876300.

97. Cheng G, Muench SP, Zhou Y, Afanador GA, Mui EJ, Fomovska A, Hickman MR. Design, synthesis, and biological activity of diaryl ether inhibitors of Toxoplasma gondii enoyl reductase. Bioorganic \& Medicinal Chemistry Letters. 2013;23(7):2035-2043.

(c) 2019 Sharif et al.; This is an Open Access article distributed under the terms of the Creative Commons Attribution License (http://creativecommons.org/licenses/by/4.0), which permits unrestricted use, distribution, and reproduction in any medium, provided the original work is properly cited.

Peer-review history:

The peer review history for this paper can be accessed here: http://www.sdiarticle3.com/review-history/49444 